\title{
EDITORIAL 14:4 PHOTOTHERAPY ESSENTIALS
}

\author{
Toshio Ohshiro MD PhD \\ Japan Medical Laser Laboratory, Tokyo, Japan
}

Dear Colleagues, Dear Friends,

\section{Cellular Photocontrol}

It is very interesting to see how the focus in phototherapy has at last shifted round to the cell as the primary target. That has always been my contention, however, since I first became interested in the athermal photobiomodulatory effects of laser energy in the late 1970's. In fact, it has now been almost 15 years since I first tentatively put forward my 'mother computer' theory (Ohshiro T [1991]: The body computer hypothesis. In Low ReactiveLevel Laser Therapy: Practical Application. John Wiley \& Sons, Chichester. 23-24). The importance of photomodulation of the immune system; the action of laser energy on fibroblast proliferation and fibroblast-myofibroblast transformation; the photobiomodulation of macrophage response and their release of fibroblast growth factors; the action of laser energy on nerve cell conduction rates and myelin repair: these are only a few examples of important studies which have appeared during this time. They all have one thing in common, however, in that they involve photobiomodulatory changes which can affect cellular control.

My 'mother computer' theory was based on the concept of understanding the control exercised by the brain over the body, composed of larger anatomical units which were themselves subdivided into discrete blocks made up of individual cells. Each subgroup, from cell to tissue block to body, was compared to a large network of computers running in a complex system of clients with the brain as the main server or 'mother computer'. When in a normal state, the entire system works in harmony with constant positive feedback and communication between all elements and between them and the brain. When this communication is broken or the feedback loop is altered, there is an abnormal situation which we can interpret as, for example, disease or pain. I have had time in the intervening years between then and now to consider this hypothesis in more detail.

The single cell is a complete system in its own right, with its own central control, the nucleus, and cytoplasmic organelles working together to maintain the cell's energy levels and fulfill its particular function. When light energy is incident on cells of the one type in vitro, there is no doubt that, through absorption with athermal energy transfer, the photon energy can modulate the action potential of these single cells in a given way depending on such factors as wavelength and dose. Most living tissue is not composed of a single cell type, however. In the case of two cell types existing together in a single culture, one of these will usually appear as the dominant type, and this dominance can be enhanced and spread to the culture environment by photobiomodulation with a laser or other noncoherent light source.

In the case of the whole body organism consideration, it is possible to exercise control over certain nerve system cells as a result of which the blood supply to the central processing unit, the brain, is enhanced: I have referred to this as 'cerebral revitalization', and this is the reason why I advocate always starting laser therapy at the Atlas/C2 intervertebral space and working round the skull base, no matter what pain or disease entity is being treated. I believe that with this consideration I can achieve a 'preactivation' with enhanced cerebral blood flow to the control centres which can restore normality to an abnormal situation. By activating the CPU first in cerebral revitalization the various channels of communication are opened: by then working from the proximal channels (blood, lymphatic and neural) distally towards the point of injury or disease in my proximal priority method, it is quite often the case that by the time I come to irradiate points around the area of interest the pain may well already have been at least attenuated as the proximal lines of communication have been opened. 
It can thus be argued that in the living body consideration, the brain (CPU) is the most important component. Without constant two-way communication and positive feedback between the brain and the body the organism will become uncoordinated which can lead in extreme cases to a panic situation, and certainly contributes to the development of harmful 'vicious circles' where there is a self-feeding negative feedback loop in which a painful situation, for example, becomes more and more painful because there is no communication between the area of interest and the body's CPU. In these situations if we first of all treat only the area of interest the pain can be exacerbated because we are not approaching the problem from the point of view of restoring contact with the CPU, and this can lead to undesirable side effects.

From a clinical consideration, this approach is essential when considering the improvement of our patients' quality of life. From the research standpoint, the same approach can now be seen in the increasing applications of and research into molecular biology and specific wavelength considerations, such as recent developments in applications of the excimer laser in molecular biology.

When we consider terrestrial sunlight with its vast range of wavebands from near UV to far IR, from work by Karu and others it is obvious that there are antagonists and protagonists, that certain wavelengths result in an amplified synergistic effect while others cancel each other out or even provide retardative effects. It is no longer enough to consider the photoresponse of a single cell to a single wavelength, but we have to investigate further the many wavelength-specific photoreceptors which can exist even in a single cell, and how these help certain cell types to become dominant over others, for example the increased phagocytic and chemotaxic action of neutrophils to invading pathogens in response to irradiation with near IR light. We must all pool our resources, and then perhaps we can finally unravel some of the mysteries of cellular photocontrol.

\section{International Phototherapy Association (IPTA)}

In its way, I strongly believe that the IPTA is another 'phototherapy essential'! As I announced in the Editorial to 14:3, the IPTA was formed in Tokyo during the September 2005 16th congress of the International Society for Laser Surgery and Medicine (ISLSM) and the first meeting of the
World Federation of Societies for Laser Medicine and Surgery (WFSLMS). Then it was a tentative thought but the thought is now reality. Membership applications have started to appear, and the first IPTA meeting is firmly set to be held in Suwa City in the beautiful Japanese Alps on July 1st and 2nd 2006, in tandem with the Japan Laser Therapy Association (JaLTA) annual congress. Thereafter, IPTA congresses will be held in odd years starting with the second congress in the USA in 2007, as the World Association for Laser Therapy (WALT) holds its meetings in even years. Despite this, we felt it was essential to get the first IPTA meeting underway next year even though it is an even year.

The IPTA Executive is as follows:

\section{Honorary Presidents:}

Prof. Kazuhiko Atsumi (Tokyo, Japan) [confirmed] Prof. Isaac Kaplan (Tel Aviv, Israel) [tentative]

\section{President}

Dr Yoshimi Asagai (Suwa City, Japan) [confirmed]

Secretary-General

Prof David Baxter (Otago, New Zealand) [confirmed]

\section{Treasurer}

Prof Toshio Ohshiro (Tokyo, Japan) [confirmed]

\section{President-Elect}

Prof Ronald Wheeland (Arizona, USA) [confirmed]

The First Announcement for the first IPTA congress will be on its way to you soon, and will also be available on the IPTA website which is now under construction. Please see pages 191 and 192 in this issue for an explanatory sheet about IPTA, and an IPTA membership application form. Remember that, as part of your membership entitlement as an IPTA member, you will receive 4 issues a year of Laser Therapy, which has been recognized as the official IPTA journal.

The first IPTA congress will have two main sessions on the 1st and 2nd of July comprising keynote lectures and round table symposia, starting at 07:00 and finishing at 14:00, with a bus tour to the beautiful highland resort of Kamikouchi on the afternoon of the 1st, featuring breath-taking scenery and bubbling hot springs, and free time to stroll gently round the lovely environs of Suwa City on the afternoon of the 2nd. Don't miss this fantastic meeting!

Some may see IPTA as a rival to WALT. In 
fact, far from setting up as a rival to WALT, we hope that IPTA and WALT will work together as a strong team, to advance the tenets of laser therapy, phototherapy and photobiomodulation, with phototherapy embracing all aspects of the therapeutic use of light, including photodynamic therapy (PDT). As an international champion of the latter, we are pleased to welcome Prof Harubami Kato to the Laser Therapy editorial board. I would like to confirm that all members of the Board have been approached for their written confirmation of their willingness to serve on the Board, and all will become IPTA members as part of their commitment to what Laser Therapy and the IPTA stand for.

So, as we move forward into 2006, the Year of the Dog in the Oriental 12 animal Zodiac, it only remains for me, on behalf of the entire Laser Therapy Editorial and production staff, to wish you all a bright and phototherapeutic New Year ...... join the IPTA, and help make it even brighter!!

Tokyo, December 2005. 